November 2012

\title{
Sternal pain after rigid fixation: a pilot study of randomization rigid vs conventional wire closure.
}

\author{
Hitoshi Hirose \\ Thomas Jefferson University \\ Hong Mun Kong \\ Thomas Jefferson University \\ Shigeki Tabata \\ Thomas Jefferson University \\ Kentaro Yamane \\ Thomas Jefferson University \\ Margaret Lusardi \\ Thomas Jefferson University
}

Follow this and additional works at: https://jdc.jefferson.edu/surgeryfp See next page for additional authors

Part of the Surgery Commons

\section{Let us know how access to this document benefits you}

\section{Recommended Citation}

Kong HM, Tabata S, Yamane K, Lusardi, M, Bogar L, Guerraty A, Diehl JT, Hirose H. Sternal pain after rigid fixation: a pilot study of randomization rigid vs conventional wire closure. Presented at Chest 2012, Atlanta GA, October 20-25.

This Article is brought to you for free and open access by the Jefferson Digital Commons. The Jefferson Digital Commons is a service of Thomas Jefferson University's Center for Teaching and Learning (CTL). The Commons is a showcase for Jefferson books and journals, peer-reviewed scholarly publications, unique historical collections from the University archives, and teaching tools. The Jefferson Digital Commons allows researchers and interested readers anywhere in the world to learn about and keep up to date with Jefferson scholarship. This article has been accepted for inclusion in Department of Surgery Faculty Papers by an authorized administrator of the Jefferson Digital Commons. For more information, please contact: JeffersonDigitalCommons@jefferson.edu. 


\section{Authors}

Hitoshi Hirose, Hong Mun Kong, Shigeki Tabata, Kentaro Yamane, Margaret Lusardi, Linda J. Bogar, and James Diehl 
Jefferson. Sternal pain after rigid fixation: a pilot study of randomization rigid vs conventional wire closure.

Hong Mun Kong, BS, Shigeki Tabata, MD, Kentaro Yamane, MD, Margaret Lusardi RN, Linda Bogar, MD, James T. Diehl, MD, Hitoshi Hirose, MD From Thomas Jefferson University Hospital, Philadelphia, PA, USA.

\section{Objective}

To investigate if rigid closure reduces sternal pain
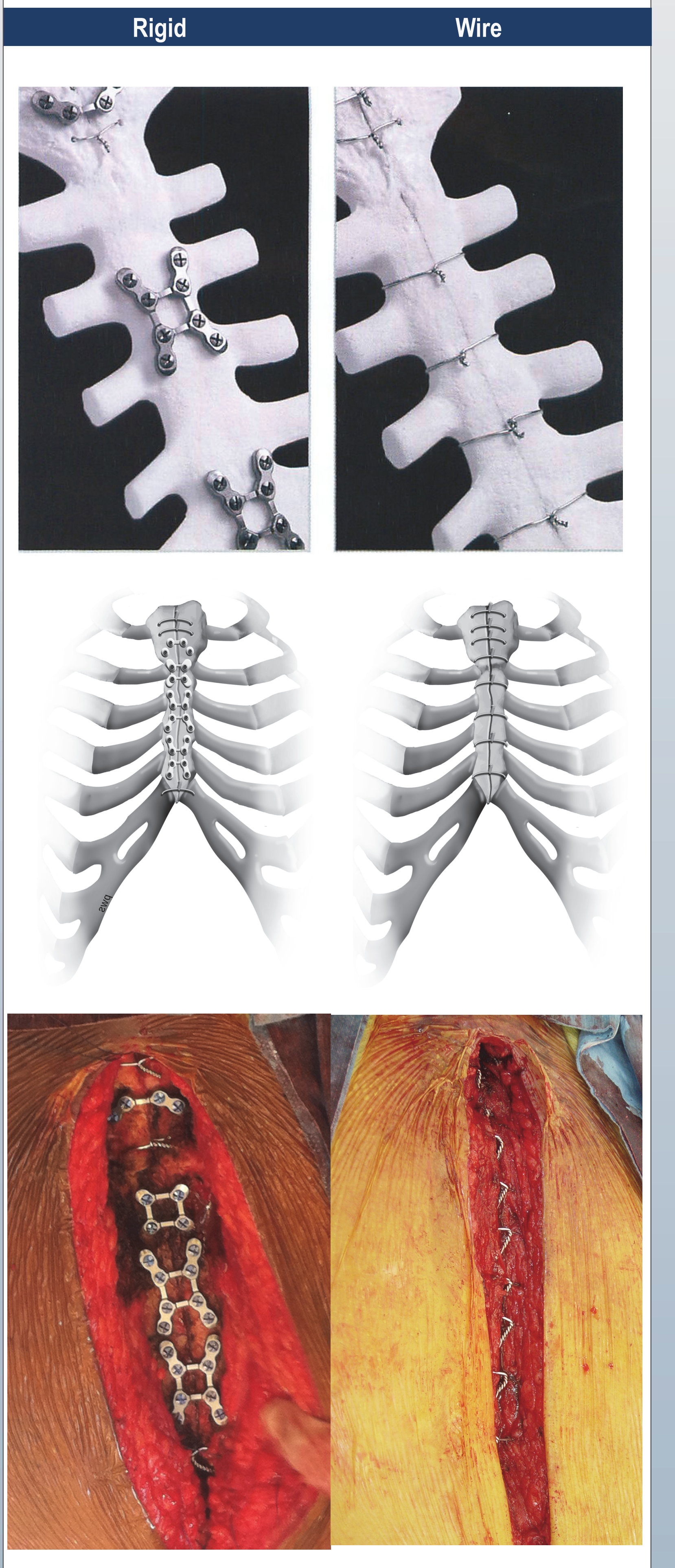

\section{Methods}

Prospective randomized CABG +/- valve Study period: 07/2011 - 1/2012

Rigid fixation: $\mathrm{n}=11$

Wire closure: $\mathrm{n}=15$

\begin{tabular}{|c|c|c|c|c|c|}
\hline \multicolumn{2}{|c|}{ Methods } & \multicolumn{4}{|c|}{ Postop Outcomes } \\
\hline \multicolumn{2}{|c|}{$\begin{array}{l}\text { Prospective randomized CABG }+/ \text { - valve } \\
\text { Study period: } 07 / 2011-1 / 2012 \\
\text { Rigid fixation: } n=11 \\
\text { Wire closure: } n=15\end{array}$} & $\begin{array}{l}\text { Intubation hours } \\
\text { Intubation }>24 \text { h }\end{array}$ & $\begin{array}{l}\text { Rigid } \\
7.3 \pm 3.1 \\
0\end{array}$ & $\begin{array}{l}\text { Wire } \\
9.2 \pm 7.2 \\
1(6.7 \%)\end{array}$ & $\begin{array}{l}\mathbf{P} \\
0.37 \\
0.38\end{array}$ \\
\hline \multicolumn{2}{|c|}{ Pre-Op and Intra-Op Exclusions } & ICU stay hours & $55 \pm 34$ & $41 \pm 24$ & 0.26 \\
\hline $\begin{array}{l}\text { Preop exclusions (78) } \\
\text { Age >80 (14) } \\
\text { Emergency (6) } \\
\text { Redo sternum (11) } \\
\text { Hemodialysis (8) } \\
\text { Hx of Osteoporosis (5) } \\
\text { Radiation hx (1) } \\
\text { Malignancy (5) } \\
\text { Immunosuppression (2) } \\
\text { Known coagulopathy (2) } \\
\text { Infections, IE (5) } \\
\text { Metal allergy (1) }\end{array}$ & $\begin{array}{l}\text { Intraop exclusions (10) } \\
\text { Unexpected aortic } \\
\text { surgery (1) } \\
\text { Osteoporosis (4) } \\
\text { Bleeding (5) }\end{array}$ & $\begin{array}{l}\text { Postop CVA } \\
\text { Atrial Fibrillation } \\
\text { Superf sternal infection } \\
\text { Deep sternal infection } \\
\text { Pneumonia }\end{array}$ & $\begin{array}{l}1(9 \%) \\
4(36 \%) \\
0 \\
1(9 \%) \\
0 \\
\text { in Score }\end{array}$ & $\begin{array}{l}0 \\
6(40 \%) \\
1(7 \%) \\
0 \\
0\end{array}$ & $\begin{array}{l}0.23 \\
0.85 \\
0.38 \\
0.23 \\
0.99\end{array}$ \\
\hline
\end{tabular}

BMl above $40(4)$

Compliance (4) Refusal (10)

\begin{tabular}{|c|c|c|c|}
\hline $\begin{array}{l}\text { Total number of CABG +/- valve } \\
\mathrm{N}=113\end{array}$ & \multicolumn{3}{|c|}{ Randomization } \\
\hline \multicolumn{4}{|c|}{ Preop exclusions (78) } \\
\hline \multicolumn{4}{|c|}{ Intraop exclusions (10) } \\
\hline \multicolumn{4}{|c|}{$\begin{array}{l}\begin{array}{l}\text { Total number of Randomization } \\
\mathrm{N}=26\end{array} \\
\end{array}$} \\
\hline \multicolumn{2}{|c|}{ Rigid Fixation (11) } & \multicolumn{2}{|c|}{ Wire Closure (15) } \\
\hline \multicolumn{4}{|c|}{ Patient Risk Factors } \\
\hline & Rigid & Wire & $\mathbf{P}$ \\
\hline Age & $67 \pm 8$ & $66 \pm 10$ & 0.78 \\
\hline Male & $10(91 \%)$ & $13(87 \%)$ & 0.74 \\
\hline BMI & $30 \pm 6$ & $28 \pm 5$ & 0.30 \\
\hline Poor EF(<40\%) & $1(9.1 \%)$ & 0 & 0.23 \\
\hline Diabetes & $6(55 \%)$ & $5(33 \%)$ & 0.28 \\
\hline Insulin user & $2(18 \%)$ & $4(27 \%)$ & 0.61 \\
\hline Smoking & $5(46 \%)$ & $6(40 \%)$ & 0.78 \\
\hline PVD & $1(9.1 \%)$ & 0 & 0.23 \\
\hline Cr above 1.5 & $1(9.1 \%)$ & 0 & 0.23 \\
\hline CABG & $8(73 \%)$ & $13(87 \%)$ & 0.37 \\
\hline Valve & $5(46 \%)$ & $4(27 \%)$ & 0.32 \\
\hline CABG + Valve & $2(18 \%)$ & $2(13 \%)$ & 0.74 \\
\hline
\end{tabular}

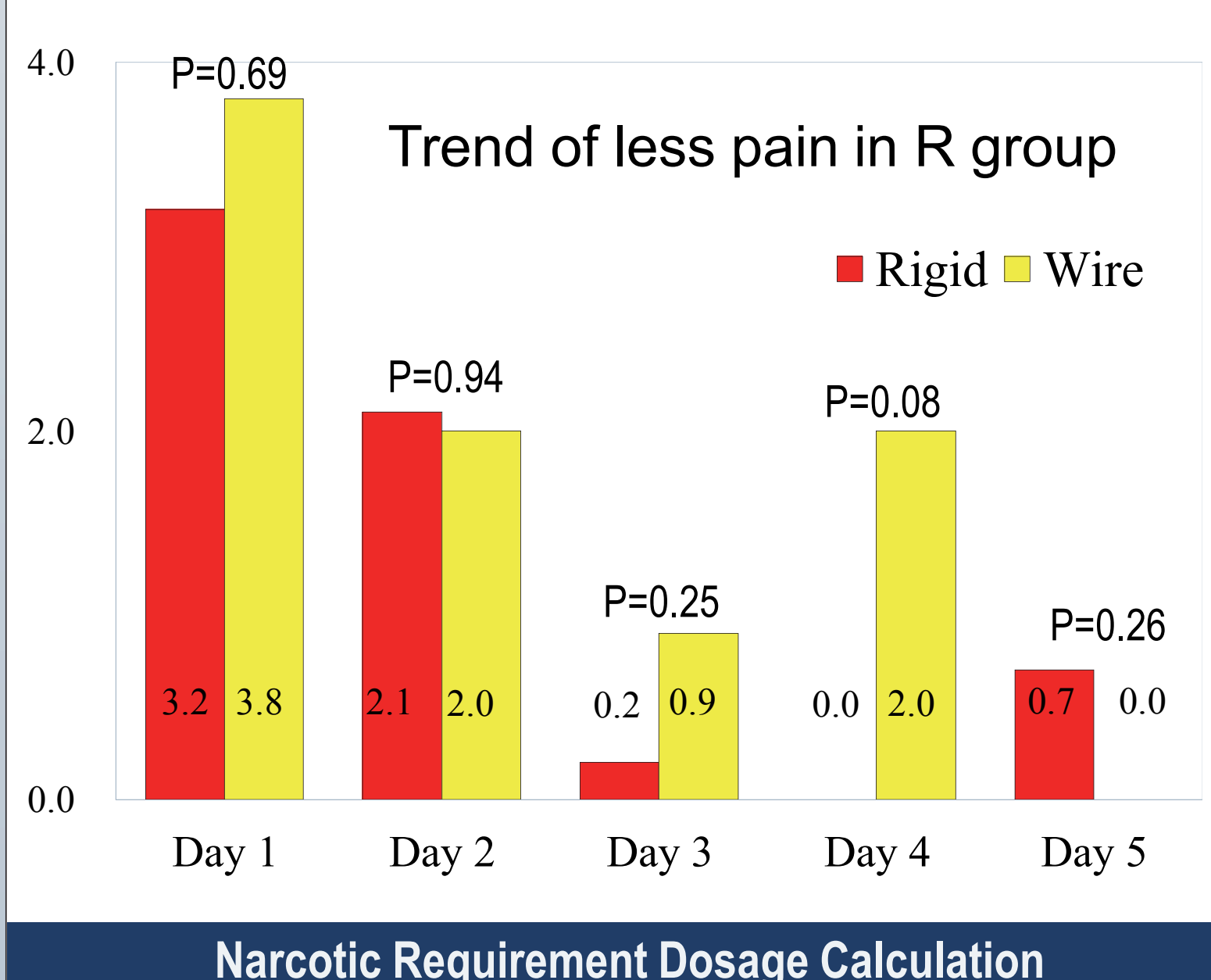

24 hours narcotic requirement was calculated using the following formula and expressed in IV morphine equivalent

$\begin{array}{lll} & & \\ \text { Morphine } & \text { IV } & \text { PO } \\ \text { Hydromorphone } & 0.15 \mathrm{mg} & 3 \mathrm{mg} \\ \text { Percocet } & \text { N/A } & 0.75 \mathrm{mg} \\ \text { Fentanyl } & 0.01 \mathrm{mg}(10 \mathrm{mcg}) & \text { N/A }\end{array}$

e.g.

$5 \mathrm{mg} P \mathrm{PO}$ morphine is equianalgesic to $1.33 \mathrm{mg} \mathrm{IV}$ morphine.

$2 \mathrm{mg} P O$ hydromorphone is equianalgesic to $2.67 \mathrm{mg}$ IV morphine

Adapted from: Debria B. et al. Opioid equianalgesic calculations. J Palliabive Med. 1999; 2: 209-218.

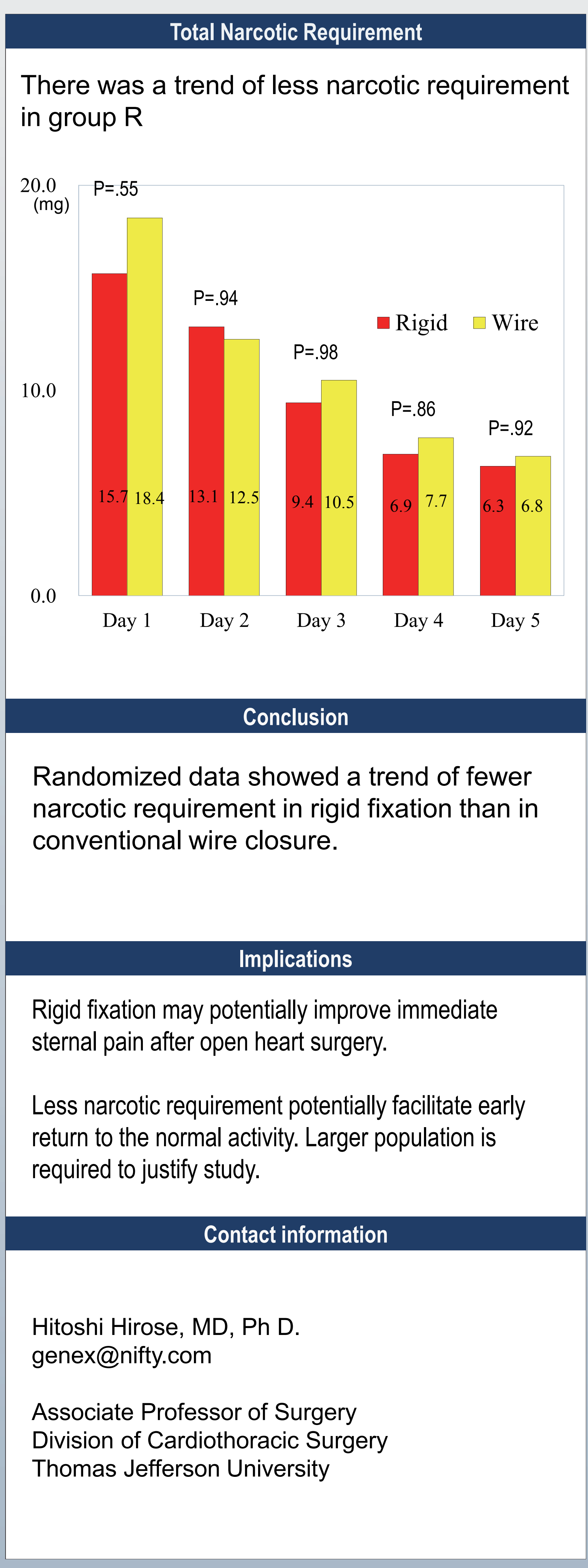

